\title{
A Turning Point in Alzheimer's Research: Harmonized Research Strategies and Novel Investments in Public Health Infrastructure Are Reenergizing the Field, and Rekindling Hope for Those Affected by Alzheimer's and Related Dementias
}

\author{
M.C. Carrillo ${ }^{1}$, H.M. Snyder ${ }^{1}$, R. Conant ${ }^{2}$, S. Worley ${ }^{3}$, R. Egge $e^{2}$

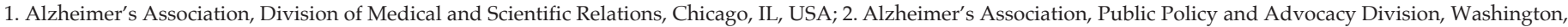 \\ DC, USA; 3. Independent Science Writer, Devon, PA, USA

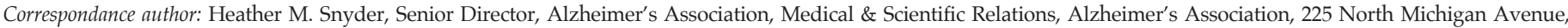 \\ Suite 1800, Chicago, Illinois 60601, USA, hsnyder@alz.org \\ J Prev Alz Dis 2019;4(6):214-216 \\ Published online September 19, 2019, http:/ / dx.doi.org/10.14283/jpad.2019.36
}

\section{A} lzheimer's disease (AD) and related dementias (ADRD) are complex global health issues that require resources and commitments from around the world. The international research community continues to build upon knowledge and generate fresh ideas and strategies to move toward an effective therapy to treat, delay, or prevent ADRD. With accelerated momentum and more funding, the field is poised to hasten the discovery of interventions to stop, slow, or prevent disease progression, and improve care and quality of life for those affected.

\section{The urgent need for interventions}

An estimated 50 million people worldwide are currently living with dementia-a number that is expected to grow to 82 million by 2030, and more than triple to approximately 152 million by 2050 (1). The worldwide estimated cost of dementia is approximately US $\$ 1$ trillion, a figure that will double to approximately US\$ 2 trillion by 2030 (1). A crisis of this magnitude requires significant commitment and investments. In the US, the National Institutes of Health (NIH) is leading the way with $\$ 2.3$ billion in funding for ADRD research (2).

The growing awareness of the complexity of $\mathrm{AD}$ and the importance of pursuing strategies that combine multiple treatment approaches are the most impactful developments in dementia science in recent years. Combination treatment approaches include pharmacological therapies and lifestyle modifications, in order to achieve the goal of effectively treating or preventing ADRD by 2025. This knowledge is supported by accumulating evidence that the underpinnings of $\mathrm{AD}$ occur over an extended period of time (3). Advances in cancer and heart disease, including early detection and interventions, also recognize and support a multipronged approach to addressing underlying disease complexity.
While furthering our understanding of the biological underpinnings of ADRD at all stages along the disease continuum, one guiding principle will be to advance strategies similar to those used for cancer and heart disease. These strategies focus on early detection and prevention, as well as a combination of lifestyle and pharmacologic interventions. Another aim will be to place an unprecedented emphasis on coordinating and harmonizing all research efforts so that all avenues of exploration and research build upon and reinforce one another. These objectives already are reflected in current avenues of research, funding strategies and initiatives, and forthright new directions in public health infrastructure.

\section{Focus on prevention and lifestyle}

In keeping with efforts to address $\mathrm{AD}$ at all stages along the disease continuum, the field has evolved toward placing a more intense focus on prevention. This focus has led to the design and launch of several revolutionary secondary prevention trials during the past seven years, which are targeting underlying pathophysiology in individuals at risk for $\mathrm{AD}$ with the goal of preventing AD symptoms. Among the largest secondary AD prevention trials currently underway are the Alzheimer's Prevention Initiative (API) Autosomal-Dominant AD, API APOE4 trial, Dominantly Inherited Alzheimer Network Trials Unit, and AntiAmyloid Treatment in Asymptomatic Alzheimer's Disease/Longitudinal Evaluation of Amyloid Risk and Neurodegeneration trials (4). With an emphasis on coordinating and harmonizing research efforts, FBRI and the Alzheimer's Association lead the Collaboration for Alzheimer's Prevention, which functions to encourage regular dialogue among the prevention studies regarding all aspects of study design and outcomes. As the field reaches the midpoint of 2019, these trials are maturing, 
and lessons from their launch and progress are informing the next generation of clinical studies and paving the way for improved clinical and diagnostic decision making.

The AD field remains firmly committed to examining and better understanding the role of multiple lifestyle factors in the development and prevention of $A D$, based on growing evidence that exercise, education, complexity of occupation, and other lifestyle factors may have protective effects for those at risk for dementia. Among the most intriguing findings in this area to date have been those from the Finnish Geriatric Intervention Study to Prevent Cognitive Impairment and Disability (FINGER) Study. This large-scale two-year study, which demonstrated that combination therapy comprising physical exercise, nutritional modification, cognitive stimulation, and self-monitoring of heart health risk factors had a protective effect on cognitive function, has ignited hope and inspired the launch of similar trials around the world to determine whether such factors might also be preventive for dementia.

In 2019, recruitment is now underway for the largest of these trials, the U.S. Study to Protect Brain Health Through Lifestyle Intervention to Reduce Risk (U.S. POINTER). U.S. POINTER, led and funded by the Alzheimer's Association, is a two-year clinical trial to evaluate whether lifestyle interventions that simultaneously target multiple behavioral modifications protect cognitive function in older adults at increased risk for cognitive decline. U.S. POINTER will enroll approximately 2,000 older volunteers (ages 60-79) from five large health care networks across the United States. Community partners, including the national network of Alzheimer's Association Chapters, will assist with intervention delivery and in turn set the stage for an accessible and sustainable community-based model for strategies to reduce risk. Two lifestyle interventions will be compared to determine whether cognitive benefits from a structured program differ from those of a selfguided program.

U.S. POINTER and FINGER are part of a global network, bringing together initiatives designed to evaluate multimodal lifestyle interventions. This network Worldwide FINGERS (WW-FINGERS), convenes annually virtually and in person with aims of sharing experiences, harmonizing data, and planning joint international initiatives for the prevention of cognitive impairment or dementia.

\section{A BOLD initiative}

While the research community is devoted to advancing potential interventions to stop, slow, or prevent disease progression, there are millions affected worldwide, including more than 5.8 million Americans, living with Alzheimer's today. There continues to be a significant unmet need for improved quality of life for those already living with $\mathrm{AD}$ and their caregivers. To help reduce some of the burdensome costs associated with $\mathrm{AD}$, the field has recognized that it is essential to invest not only in basic and clinical research but also in an international public health response to $\mathrm{AD}$, with a focus on comprehensive disease assessment, monitoring and care.

A milestone in meeting these needs in the US, the Building Our Largest Dementia (BOLD) Infrastructure for Alzheimer's Act-legislation designed to create a vital public health infrastructure to address AD-was signed into law on December 31, 2018. The passage of this historic act, which attracted enormous bipartisan support and demonstrated that Congress remains fully committed to the fight against Alzheimer's disease, represents a critical step toward addressing the AD public health crisis. The new law will lead to the establishment of Alzheimer's public health centers of excellence across the US, provide funding to public health departments to implement effective Alzheimer's interventions, and increase analysis and timely reporting of data on cognitive decline and caregiving to inform future public health initiatives and improve measures of progress.

The BOLD Infrastructure for Alzheimer's Act was originally developed and shepherded into law by the Alzheimer's Impact Movement (AIM), the advocacy arm of the Alzheimer's Association. AIM will also help to further existing policies designed to strengthen the US response to AD. In 2005, for example, the Alzheimer's Association partnered with the Centers for Disease Control and Prevention to create and launch the Healthy Brain Initiative (HBI) and Public Health Road Maps. The HBI Road Maps feature strategic actions that state and local public health departments can take to address cognitive impairment, promote improved cognitive functioning, and help meet the needs of caregivers. The passage of the BOLD Infrastructure for Alzheimer's Act will enhance implementation of the HBI Road Maps by promoting early detection and diagnosis, reducing lifestyle-related risks, and preventing avoidable hospitalizations.

\section{Looking ahead}

As the world's largest nonprofit funder of Alzheimer's research, and as the nonprofit with the highest impact worldwide in Alzheimer's and dementia science, the Alzheimer's Association is currently investing over $\$ 165$ million in more than 450 best-of-field active projects in 25 countries. Through policy and research initiatives, the Alzheimer's Association continues its mission of serving as both a leader and a catalyst in funding, as well as orchestrating a broad range of research initiatives. In our role as a global convener, we are actively pursuing opportunities to expand public and private investments in $\mathrm{AD}$ research, advance the proliferation of potential therapeutic targets, launch new clinical trials to test these interventions, and design novel studies to help us better understand risks for dementia as well as the best 
approaches to clinical and long-term care.

Research investments in the biological underpinnings of the disease (often referred to as basic research) continue to be a top priority. AIM has been instrumental in securing increased federal funding at unprecedented levels to support critical basic and translational science research by the NIH, which leads the nation in biomedical research on ADRD. Recent efforts by AIM have more than quadrupled Alzheimer's research funding at the NIH since the passage of the National Alzheimer's Project Act, and have led to an historic \$425 million increase for Alzheimer's research at the NIH for fiscal year 2019.

Increased federal funding for the NIH has come at a critical moment. Many of the late-stage clinical trials today were designed during a time when funding for research into the biological underpinnings of the disease was inadequate. Recent phase 3 studies have not yielded the results that are desperately needed: more effective treatments for Alzheimer's dementia. Today, many companies are changing the way that they invest in and develop future drugs including moving toward venture capital approaches to partnerships and licensing and outsourcing research in lieu of their own in-house research. Increased dollars in the field for discovery of innovative and diverse mechanisms and targets is more critical than ever to enable the development of future treatments from the bottom up, from novel ideas through biotech early development and beyond. With these vital boosts in NIH funding, scientists are able to work at a more rapid pace to advance basic disease knowledge, explore ways to reduce risk of dementia, discover new biomarkers for early diagnosis and drug targeting, and develop potential treatments.

As we move forward at a rapid pace, the coordination and harmonization of all initiatives will remain the key to success for finding prevention and treatment options. In all of our endeavors-whether we are working toward a common language or nomenclature for generating and testing hypotheses, or establishing common measures of neurodegeneration and neuronal injury - the AD community will continue to thrive as long as we remain committed to unifying our efforts (5). With continued persistence and dedication, careful orchestration of initiatives will sustain our focus on developing multipronged treatment strategies over the $\mathrm{AD}$ continuum and will greatly improve our likelihood of achieving effective treatment and prevention of ADRD.

Disclosures: MC Carrillo, HM Snyder, R Conant and R Egge are employees of the Alzheimer's Association.

Open Access: This article is distributed under the terms of the Creative Commons Attribution 4.0 International License (http:/ / creativecommons.org/ licenses/by/4.0/), which permits use, duplication, adaptation, distribution and reproduction in any medium or format, as long as you give appropriate credit to the original author(s) and the source, provide a link to the Creative Commons license and indicate if changes were made.

\section{References}

1. Alzheimer's Disease International. World Alzheimer Report 2018. Available from: https://www.alz.co.uk/research/world-report. Accessed: 17 April 2019.

2. National Institutes of Health. Open science, big data, and you: working together to treat and prevent Alzheimer's disease and related dementiasbypass budget 2020. Available at: https://www.nia.nih.gov/sites/default/ files / 2018-07 / fy2020-bypass-budgetreport-final.pdf. Accessed April 17, 2019

3. Jack CR, Jr, Knopman DS, Jagust WJ, et al. Hypothetical model of dynamic biomarkers of the Alzheimer's pathological cascade. Lancet Neurol. 2010;9:119-128.

4. Hsu DC, Marshall GA. Primary and secondary prevention trials in Alzheimer disease: Looking back, moving forward. Curr Alzheimer Res. 2017;14(4):426440

5. Jack C, Bennett D, Blennow K, et al. NIA-AA Research Framework: Toward a biological definition of Alzheimer's disease .Alzheimer's Dement. 2018;14(4): $535-562$. 